8 weeks. The treatment was assessed using bladder diaries, quality-of-life surveys, urodynamic studies and one-hour pad weight testing.

In the urge incontinence group, 17 (85.0\%) patients were cured or improved (defined as frequency of incontinence decreasing by $>50 \%$ ) and improvements were maintained 24 weeks after the last treatment in 9 (52.7\%) of these cases. In the stress incontinence group, a complete cure or an improvement was observed in 16 (94.0\%) patients, of whom $13(81.3 \%)$ maintained their improvement at 24 weeks. Quality-of-life (I-QOL) scores at 4 weeks were significantly increased compared to baseline in both patient groups.

The authors conclude that ExMI is useful for treating urge incontinence, although stress incontinence patients showed higher response rates in this study. Further studies are needed to confirm these results and to optimize the treatment parameters.

Original article Yokoyama T et al. (2004) Extracorporeal magnetic innervation treatment for urinary incontinence. Int J Urol 11: 602-606

\section{VUR resolved by bladder augmentation without antireflux surgery}

Children with neurogenic bladder, exstrophyepispadias complex or infravesical obstruction commonly undergo bladder augmentation. In addition, vesico-ureteric reflux (VUR) is sometimes treated by antireflux surgery in these patients. It is unclear, however, whether antireflux surgery is necessary, since VUR may resolve as a result of the augmentation process. Soylet et al. have carried out a retrospective study to address this question.

The authors studied the records of 38 children with neurogenic bladder, exstrophyepispadias complex or infravesical obstruction who had undergone bladder augmentation. VUR had been detected in all patients before surgery. Group 1 patients $(n=15)$ had no antireflux surgery, whereas children in Group $2(n=23)$ underwent ureteric reimplantation, either at the same time as the cystoplasty or afterwards.

VUR resolved after cystoplasty in $97 \%$ and $93 \%$ of refluxing renal units in Groups 1 and 2, respectively. The expected bladder capacities increased following surgery, from $35 \%$ to $86 \%$ in Group 1 and from 38\% to 90\% in Group 2.

Renal function was maintained in both groups during the mean follow-up of 43 months (range 12-84 months).

Concluding that antireflux surgery had no significant effect on the resolution of VUR or on renal function in this study, the authors recommend using augmentation cystoplasty alone in children with neurogenic bladder, exstrophyepispadias complex or infravesical obstruction and high- or low-grade VUR.

Original article Soylet Y et al. (2004) Quo vadis? Ureteric reimplantation or ignoring reflux during augmentation cystoplasty. BJU Int 94: 379-380

\title{
Plant-derived medication for LUTS
}

EBM

A lipido-sterolic extract of the American dwarf palm tree Serenoa repens is increasingly used to treat lower urinary tract symptoms (LUTS) associated with benign prostatic hyperplasia (BPH). This approach is thought to alleviate LUTS without producing the negative effectsin particular the impact on sexual functionassociated with more traditional therapies such as $\alpha$-blockers and $5 \alpha$-reductase inhibitors. Gerber and Fitzpatrick have reviewed the clinical data available on S. repens extract to assess its potential in patients with BPH.

The search was restricted to clinical trials of Permixon ${ }^{\circledR}$ (Pierre Fabre Médicament, Castres, France), the best studied and most common of several brands of S. repens extract. The authors identified 18 individual studies and 2 meta-analyses, with outcomes typically including prostatic symptoms, daytime and nocturnal urinary frequency and flow, PSA levels, quality of life and sexual function.

According to Gerber and Fitzpatrick, the clinical data indicate that Permixon produces similar results to those obtained using $\alpha$-blockers and $5 a$-reductase inhibitors and is better tolerated. Warning that the various brands of S. repens extract differ in terms of composition and consistency of formula, the authors suggest that Permixon should be considered as a first-line therapy for the treatment of LUTS in BPH.

Original article Gerber GS and Fitzpatrick JM (2004) The role of a lipido-sterolic extract of Serenoa repens in the management of lower urinary tract symptoms associated with benign prostatic hyperplasia. BJU Int 94: 338-344 